\title{
Lensless wide-field single-shot imaging through turbid media based on object-modulated speckles
}

\author{
Pengfei Wu, ${ }^{1,2}$ ZI LIANG, $^{1,2}$ XING ZhaO, ${ }^{1,2}$ LeI SU $^{3}$, LIPEI SONG ${ }^{1,2,3,4^{*}}$
}

\author{
${ }^{1}$ Institute of Modern Optics, Nankai University, Tianjin, 300350, China, \\ ${ }^{2}$ Key Laboratory of Optical Information Science and Technology of Ministry of Education, Nankai Univeristy, Tianjin, 300350,China, \\ ${ }^{3}$ School of Engineering and Materials Science, Queen Mary University of London, E1 4NS, London. United Kingdom \\ ${ }^{4}$ State Key Laboratory of Quantum Optics and Quantum Optics Devices, Institute of Opto-Electronics, Shanxi University, Taiyuan 030006, China \\ *Corresponding author:gm_imo@nankai.edu.cn,
}

The need to image objects through light-scattering materials is common in a range of applications. Different methods have been investigated to acquire the image of the objects when diffusers are presented. In this paper, we demonstrate the object reconstruction with single-shot imaging based on correlography principle and phase retrieval algorithm with coherent illumination. We prove the possibility of reconstructing positive and negative object in both transmission and reflection modes with collimated and scattered light. Formulas for calculating the size of the object from the reconstructed image are presented. We also prove that the object can be retrieved from a small section of the raw speckle image, a property similar to holography. These interesting features will open new doors for imaging applications to wider areas.

Imaging through scattering media is common practice in many areas. For example, in biomedical imaging applications, the vessels, target cells or alien tracers are covered by highly scattering skin or other types of tissues. Because of the necessity to overcome the scattering effect, many methods have been proposed over the recent few years. Spectral analysis [1, 2] or phase conjugation [3] were used to remove the scattering effect for object image reconstruction. Such methods depend on the pre-knowledge of the medium properties that have to be acquired by pre-calibration or scanning. Ghost imaging [4-7] and holographic processes [8-11] can retrieve the information of an unknown object. However, a reference beam is necessary for both of them and sometimes a calibration is needed. In-line holography, which takes the un-scattered part of the beam as the reference to avoid an extra reference beam, was also applied to imaging through scattering material[12,13]. The application of in-line holography is usually limited by the applicable object size and intensity because the portion of the un-scattered beam needs to be larger than that of the scattered beam from the object[14]. Polarization difference (PD) imaging was also applied to imaging through scattering materials. Since PD imaging utilizes the different polarization changes induced by the scattering and the object [15-17], it is necessary to detect light intensity at different polarization directions. Wave-front shaping enables imaging through visually opaque media [18-24]. The principle of wave-front shaping is an inverse scattering process by controlling the phase of light with a spatial light modulator (SLM). It relies on careful alignment of the optical system, large data sets and calculation, or scanning the object. Based on the memory effect of speckles [25], a non-invasive imaging method through a scattering medium was proposed [26, 27]. But the memory effect restricted the angular field-of-view (AFOV) of this system to only several degrees as a result of the scattering medium. The speckle contrast approach was proved successful in imaging based on the scattering effect, particularly in blood flow imaging [28-30]. However, this method is not suitable for non-moving objects. E. Edrei and G. Scarcelli recently used the shower-curtain effect and speckled illumination to reconstruct object images between two variable diffusers [31]. But this method requires a careful focusing to the speckle pattern behind the scattering medium.. Nevertheless, the imaging correlography principle [31-35] used in this method provides a potential way to overcome the restriction given by the small field of view (FOV) on the memory effect method.

Imaging correlography with laser illumination was initially used to remove the effect of atmosphere turbulence [36]. Then Idell and Goodman [33, 35] developed a model for the generation of speckle field from the object and for reconstructing the object image from the autocorrelation of the observed speckle intensity and "errorreduction" phase retrieval algorithm. The transmission model is based on the far-field criteria and the phase disturbance directly applied on the surface of the object.

In this paper, combining correlography and phase retrieval algorithm [37, 38], Goodman and Kirkpatrick's theory [39-41] on the modulation effect of the object to individual speckles, and the property that the distance for far field criterial of diffused coherent light field is much shorter than normal light, we present a lens-less imaging method for various applications. We experimentally demonstrate 
reconstructing positive and negative object in both transmission and reflection modes with collimated and scattered light. We deduce formulas for calculating the size of the object from the reconstructed image. We also prove that the object can be retrieved from a small section of the raw speckle image, a property similar to holography. A mathematical induction shows that the input for phase retrieval method can be acquired from twice Fourier transform of the speckle image, different from the traditional correlography method. The advantages of large observing angles and depth of field using this imaging method are demonstrated.

\section{PRINCIPLE}

According to the correlograhy presented by Goodman [33, 35], the image of the object through scattering material is expressed as [33, 37]:

$$
I=|F\{o(x, y) \cdot \exp (-i s(x, y))\}|^{2}=|F\{o(x, y) \cdot p(x, y)\}|^{2}
$$

where $\mathrm{F}$ denotes Fourier transform, $o(x, y)$ is the object, $s(x, y)$ is the random phase angles introduced by the diffuser, and $p(x, y)$ is $\exp (-i s(x, y))$ for simplicity. This formula was used to describe the interstellar imaging where the distance between the diffuser and the camera is long enough to satisfy Fraunhofer diffraction. However it was proved that the distance for far-field condition of the coherent light after passing through a diffuser is much shorter than normal farfield condition and even in a wavelength scale in some situations [31, 38, 39], therefore the Fraunhofer diffraction in Equation (1) is easily satisfied in our system. Therefore, the speckle pattern to some extent carries the information of the object. According to Wiener-Khintchine theorem, the Fourier transform of the image intensity can be expressed as:

$$
\begin{aligned}
|F(I)| & =\iint_{-\infty}^{\infty} o(x+\alpha, y+\beta) \cdot o^{*}(x, y) d x d y . \\
& \left|\iint_{o(x, y)} p(\xi+\alpha, \eta+\beta) p^{*}(\xi, \eta) d \xi d \eta\right| \\
& =o \succsim o\left|\iint_{o(x, y)} p(\xi+\alpha, \eta+\beta) p^{*}(\xi, \eta) d \xi d \eta\right| \\
& =\{o(x, y) \succsim o(x, y)\} \cdot \psi(x, y)
\end{aligned}
$$

where $\psi(x, y)$ is a sharply-peaked function, similar to $\delta$ function but with a less noisy background, and the modulus of which is proportional to that of the autocorrelation of $\exp (-i s(x, y))$. The asterisk * denotes the complex conjugate and the symbol $i s$ denotes correlation calculation. Then by applying another Fourier transformation, Equation (2) changes to:

$$
\begin{aligned}
|F\{|F\{I\}|\}| & =F\{\{o(x, y) \approx o(x, y)\} \cdot \xi(x, y)\} \\
& =|F\{o(x, y)\}|^{2} \otimes|F\{\xi(x, y)\}| \\
& \approx|F\{o(x, y)\}|^{2}+B
\end{aligned}
$$

where $\otimes$ denotes convolution calculation. $|F\{\xi(x, y)\}|$ is also a sharply-peaked function that has the same form as $\psi(x, y)$, leading to the background noise $\mathrm{B}$ that is added to the root square of the power spectrum of the object and can be removed by setting an appropriate threshold. Equation (3) gives us the amplitude spectrum of the Fourier transform of the object. Therefore by simply applying twice Fourier transform, the input for "error-reduction phase retrieval algorithm" is acquired to eventually reconstruct the object, the phase of the frequency spectrum is calculated using an modified algorithm based on the method originally proposed by Fienup [40] and the method for diffraction imaging proposed by Marchesini [41]. The flow chart of the object reconstruction is shown in Fig. 1.

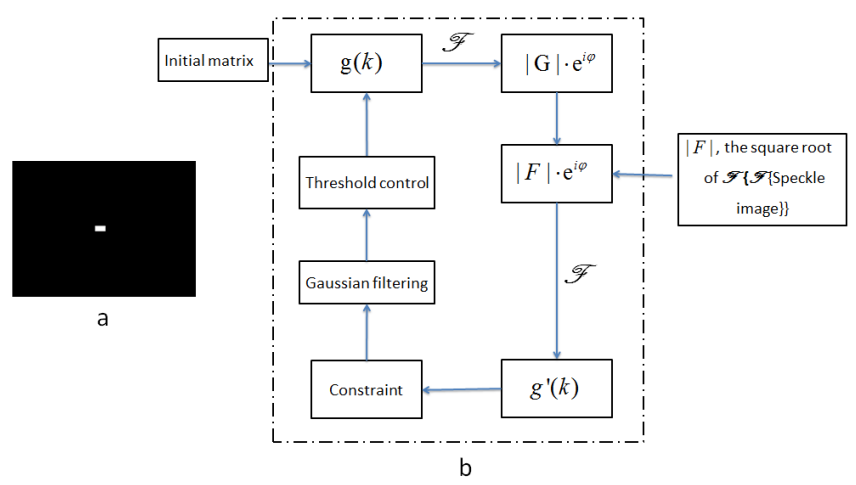

Fig. 1. (a) The initial matrix used in the iteration to generate the initial phase components. The middle white area contains $70 \times 40$ pixels. (b) Flow chart of the iteration process. The dotted line marks the iteration

loop. $F$ denotes Fourier transform, || means the absolute value, $k$ is

the iteration index.

The iteration flow follows the frame of Fienup's algorithm, but a Gaussian filter and a threshold control are introduced to the absolute value of the output image after the traditional constraint [40] is applied, as demonstrated in Fig. 1(b). By doing so, the noise level is reduced and the energy spectrum is extracted from the background. Consequently, the converging speed of the iteration can be increased.

A numerical simulation to demonstrate the imaging process is shown in Fig. 2a-i. Fig. 2a is a simple sketch of imaging through a diffuser. Fig. $2 b-c$ show the modulation effect of the aperture shape on the speckle pattern. Fig. $2 \mathrm{f}$ is the modulus of Fourier transform of Fig. $2 \mathrm{e}$, which is equal to the autocorrelation of the object multiplied by a sharply-peaked function. Here we adjusted the contrast to show the shape of the pattern. Fig. $2 \mathrm{~g}$ is the square root of the amplitude of the Fourier transform of Fig. 2f, indicating the amplitude spectrum of the object with a background noise as expressed in Equation (3). Fig. $2 \mathrm{~h}$ shows the amplitude of the Fourier transform of the object by applying a threshold to Fig. 2g. The threshold is to remove the noise and extract the amplitude spectrum of the object. The value of the threshold was 0.12 in Fig. $2 \mathrm{~g}$, where the maximum intensity was 1 (the image intensity was normalized to 1). But the proper value of the threshold varies with experiment parameters such as the sampling frequency. A robust way to determine the threshold is to apply some common image processing techniques, such as categorization or autosegmentation. Fig. $2 \mathrm{i}$ is the final reconstructed object from Fig. $2 \mathrm{~h}$ by using the phase retrieval algorithm. 


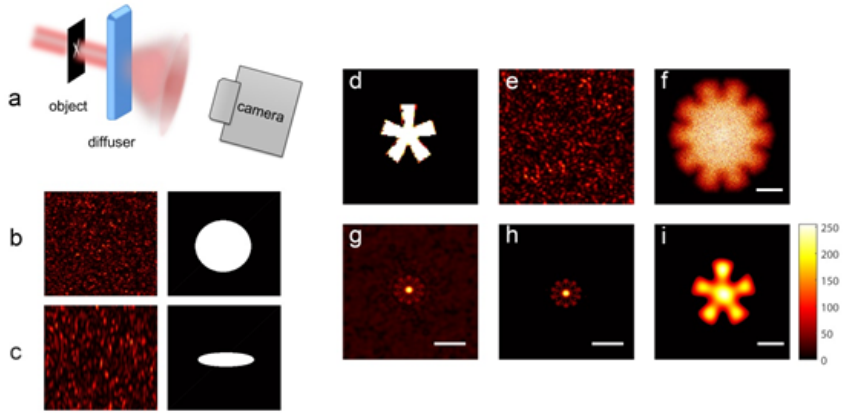

Fig. 2. Numerical simulation to show the modulation effect of the object shape on the profiles of the speckles and the image-reconstruction process. (a) A schematic of the system for imaging through a diffuser. (b)-(c) The modulation effect of the object shape (the right column) on the profiles of the speckles (the left column). (d)-(i) The complete image-reconstruction process and the output at each reconstruction step. The image reconstruction was completed with a customized MATLAB program. The object was a star-shaped aperture shown in Fig $2(d)$. A random phase distribution in a range of $[0,2 \pi]$ was added to the aperture to simulate the phase scrambling effect of the diffuser. The speckle pattern in the image plane was generated according to Equation (1) with the method described in the reference [42]. Then the image processing and the phase retrieval were applied to the synthesized speckle pattern. Scale bars are 100 pixels in (f) and (i), 30 pixels in (g) and (h). For readability, all the pictures are zoomed-in pictures. The size of the original whole images is $1000 \times 1000$ pixels. The object takes up 220 pixels. A square of $70 \times 40$ pixels with values equal to 1 was used as the initial phase input in the iteration algorithm.

\section{EXPERIMENT}

The experiment sketches are listed in the corresponding experiment sections. In the experiments, the laser beam $(\lambda=671 \mathrm{~nm})$ is collimated by a telescope system before it was directed to the first diffuser or the object. The diameter of the beam is about $1 \mathrm{~cm}$ in all the experiments except the non-invasive imaging section where the beam is a few millimeters. The speckle images are captured by a CMOS camera (Flea3-U3, Point Grey, Canada, 1280x1024, pixel size 4.8 $\mu \mathrm{m} \times 4.8 \mu \mathrm{m}$ ). The exposure time of the camera need to be adjusted to have sufficient image intensity over the camera noise but avoiding saturated pixels at the same time. In our experiments, the exposure time was changed between 20-30 ms according to the experimental setup and the size of the object. In the phase retrieval, the standard deviation of the Gaussian filter changed from 1.5 to 1 by a step of 0.025 after each 10 iterations. The threshold in our experiment was between 0.2-0.25 when the maximum pixel value was normalized to 1 .

For the positive object, also the transmission mode, the objects are positive objects and through-holes with specific shapes cut in black paperboards using laser ablation. For the negative objects, the objects are handwritten letters on normal printing paper and the illumination beam was shaped by a rectangle aperture. In the non-invasive imaging test, the object is a mirror covered by a ' $Z$ ' shaped aperture. The diffusers we used in experiment were normal white paper, ground glass and pear slices.

To satisfy Nyquist sampling criterion (speckle size should be larger than two camera pixels), the imaging distance (the distance from the camera to the diffuser) was adjusted according to the different setup of the experiments and is listed in corresponding sections. The object distance (the distance from the object to the diffuser) generally does not make much difference, as we experimentally verifies in following sections. But the object distance cannot be too long, as that may result in predominant diffraction and projection overlapping as mentioned in the Discussion Section.

We used the image processing method for diffraction imaging and but add a smooth filter before the threshold control in each iteration of the phase retrieval error-reduction algorithm inspired by Marchesini [41] (algorithms such as normal err-reduction HIO algorithm also works). A rectangle of $70 \times 40$ pixels with values equal to 1 is used as the initial input in the iteration algorithm. In all the experiments, 200 times of iteration are sufficient to obtain a solution by a standard desktop computer using a customized Matlab program.

\section{A. Imaging positive objects and size calculation}

The optical setup is shown in Fig. 3a. The objects were X-shaped through-holes cut in a piece of paper at three sizes $(2 \mathrm{~mm}, 350 \mu \mathrm{m}$, and $150 \mu \mathrm{m}$ in height respectively). A piece of normal printing paper was used as the diffuser, through which the detail of the object was blocked at room light illumination, as shown in Fig. 3b. The results of this experiment are given in Fig. 3c-e corresponding to the three different sizes of the objects respectively. In Fig. 3c-e, the left column is the raw images recorded by the camera and the right column is the reconstructed object images. The shape of the object is well reconstructed.

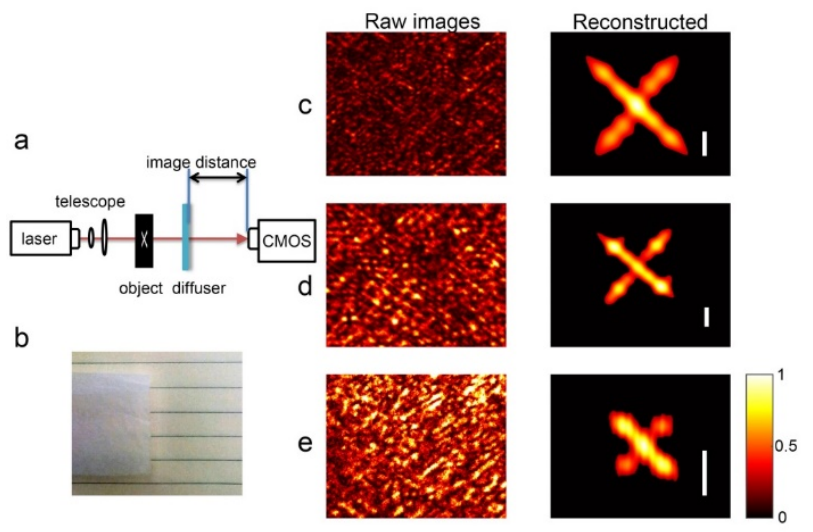

Fig. 3. Imaging objects of different dimensions. (a) light-path diagram. (b) The opaque effect of the paper. (c)-(e) The images of raw speckle patterns and the reconstructed images when the object height is $2 \mathrm{~mm}$, $350 \mu \mathrm{m}, 150 \mu \mathrm{m}$ respectively. The reconstructed images in (c)-(e) are enlarged to show the details. Scale bar is $500 \mu \mathrm{m}$ in (c) and $100 \mu \mathrm{m}$ in (d) and (e). The image distance is $10 \mathrm{~cm}$ in (c) and $2 \mathrm{~cm}$ in (d) and (e) to satisfy the criteria imposed by the Nyquist sampling frequency and the required number of speckles.

The size of the object can be calculated easily without any prior knowledge about the diffuser. The size here means the object dimension in one direction, such as length or width. It is known that the formula for calculating the speckle size is $[42,43]$ :

$$
X=\frac{\lambda \cdot Z}{H}
$$

where $\mathrm{X}$ is the speckle size, $\lambda$ is the wavelength, $\mathrm{Z}$ is the distance from the camera to the diffuser, and $\mathrm{H}$ is the size of the object. In the reconstructed image:

$$
X=\frac{L \cdot A}{N} .
$$


where $L$ is the number of pixels of the speckle image, $A$ is the size of one pixel, and $\mathrm{N}$ is the number of the pixels of the reconstructed object image [42, 43]. Since Equation (4) is equal to Equation (5), we can get:

$$
H=\frac{\lambda \cdot Z \cdot N}{L \cdot A} .
$$

In Fig. 3c, the object used is $2 \mathrm{~mm}$ in height and the image distance was $10 \mathrm{~cm}$. The reconstructed object takes 158 pixels in vertical direction. Therefore, according to Equation (6), with a 671nm laser and a pixel size of $4.8 \mu \mathrm{m}$, the height of this letter is $2.16 \mathrm{~mm}$. Similarly, we can calculate the dimension of any region of interest on the object. For example the stroke-width of the letter ' $X$ '. The precision of the calculated size can be improved by carefully measuring the distances in Equation (6). $\mathrm{L}$ and $\mathrm{N}$ are correlated and the ratio $\mathrm{L} / \mathrm{N}$ is a constant, which depends on the experiment configuration.

\section{B. Imaging negative objects}

Imaging negative objects is tested in both transmission and reflection modes. The objects are letters written on a piece of paper as shown in Fig. 4a and the optical setup is shown in Fig. 4b. By moving the camera to the position marked with the dashed line in Fig. 4b, it converts the system from the transmission mode to the reflection mode. The results are shown in Fig. 4c-e. The images in the left column in Fig. 4c-e are the raw images recorded by the camera. By using the same data processing procedures described in the theoretical section, the images of the letters are well reconstructed as shown in the last column of Fig. 4. The size of the letters can also be calculated with Equation (6).

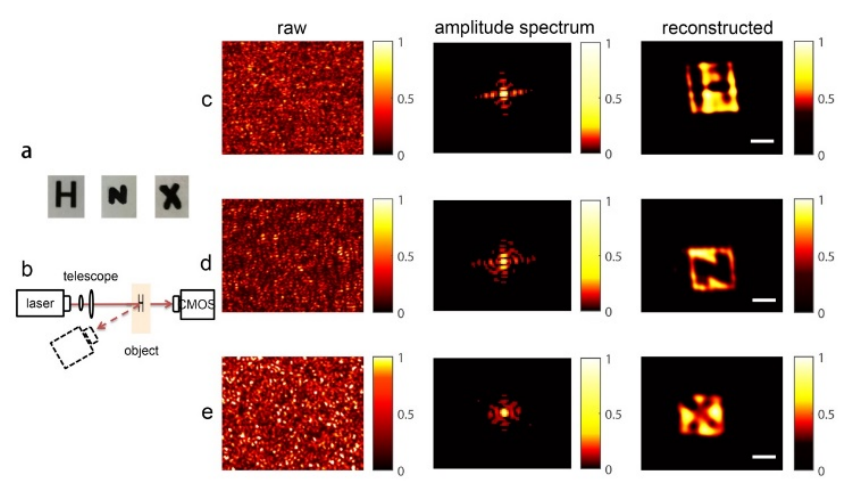

Fig. 4. Imaging negative objects. (a) The objects: letters written on a piece of paper. (b) The sketch of the optical setup. (c)-(e) Columns from the left to the right are: raw images, amplitude spectrum of the objects and the reconstructed images. (c) and (d) are the results in the transmission mode and (e) shows the result of the reflection mode. Scale bar is $2.5 \mathrm{~mm}$ and the size of the object was $5 \mathrm{~mm}$ in width. The image distance was $12 \mathrm{~cm}$.

\section{Reconstructing the object from a section of the raw speckle image}

According to Goodman and Kirkpatrick [42-44], the shape of a single speckle is modulated by the aperture shape. Furthermore, from Equation (1) we can conclude that the observed raw speckle image is a convolution of the power spectrum density (PSD) of the object (when the object is non-negative) and an ordinary speckle pattern. As a result, every single speckle in the raw image should contain the information of the object according to the property of convolution, if the aberration is negligible. Therefore, it is possible to reconstruct the object by using only a small section of the raw image. This feature allows the successful reconstruction even when sections of the speckle image are unavailable. Additionally, by reducing the image size, a spatial or temporal series of images can be recorded at different sections of the camera chip, thereby increasing the FOV, spatial resolution, or temporal resolution. Fig. 5a shows the raw speckle image captured by the camera. The white and the yellow lines divide the image into 4 and 16 sections respectively. Fig. 5b shows the reconstructed image from the top left $1 / 4$ section of the raw speckle image and Fig. $5 \mathrm{c}$ shows the reconstructed image from the top left $1 / 16$ section of the raw speckle image. In both reconstructed images, the shape and the size of the original object can be identified.
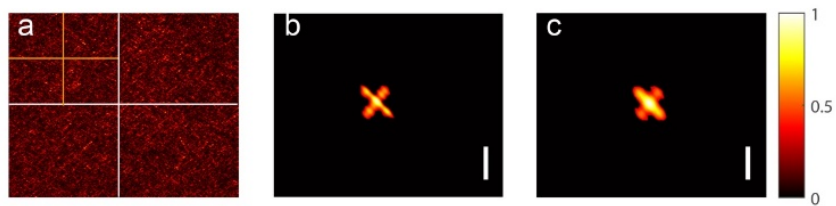

Fig. 5. Image reconstructions from a section of the raw speckle image. (a) The raw speckle image captured by the camera. (b) The reconstructed object calculated from the top left $1 / 4$ section of the speckle image. (c) The reconstructed object image calculated from $1 / 16$ section of the speckle image. Scale bar is $2 \mathrm{~mm}$, same as the object size.

Sampling frequency is important for image reconstruction. But when the speckle size is too big, which is usually true when the object is very small according to Equation (4), the image reconstruction will fail. The reason is demonstrated in Fig. 6, where high frequency components extend beyond the image border when the object size reduces from its original value in (a) to $1 / 5^{\text {th }}$ in (b) and $1 / 10^{\text {th }}$ in (c). The smaller the object is, the larger the speckles and the higher the frequencies are. As we know, the higher frequency components of the power spectrum contain the details of the object. As a result, the reconstruction cannot succeed without those high frequency components. Therefore, when this happens the speckle size need to be adjusted such as by changing the distance between the camera and the diffuser to avoid the loss of high frequency

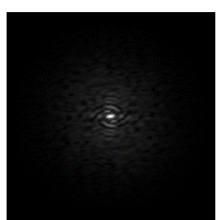

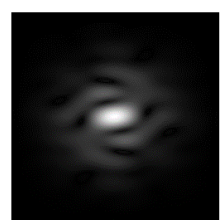

b

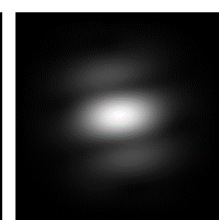

Fig.6 The power spectrum of the object when the object size reduces from the initial value (a) to $1 / 5^{\text {th }}$ in (b) and $1 / 10^{\text {th }}$ in (c).

When the section selected for the reconstruction is too small, the reconstruction may be unsuccessful. The reason is similar to that described above. The difference is that, in this situation, the speckle size or the size of PSD remains unchanged but the image size decreases. Therefore, the higher frequency components of the object may extend beyond the image border and is not included in the image reconstruction.

\section{Demonstration of the large AFOV and DOF}

Compared with imaging methods through a lens, this approach is insensitive to the observing angle. Regardless of the angular location where the raw image is taken, the quality of the reconstructed object 
images remains the same as long as the scattered light intensity at the recording location is sufficient to overcome the noise (including the shot noise and the dark current noise). But the image can be deformed due to the increasing of the observing angle. This is demonstrated in Fig. 7a-g. The diffuser was ground glass of $5 \mathrm{~mm}$ in thickness and was frosted on both sides. Fig. $7 \mathrm{a}-\mathrm{f}$ show the reconstructed object images at different observing ranging from $0^{\circ}$ to $45^{\circ}$, as shown in Fig. $7 \mathrm{~g}$. Although we only demonstrated AFOV up to $45^{\circ}$, it is expected that the AFOV can be up to any angle as long as sufficient scattered light is collected by the camera.

It should be noticed that the increase of the observing angle does introduce a certain degree of deformation to the reconstructed object image. This is similar to the situation that the image becomes thinner or shorter at an increased perspective angle. This is because the effective object shape that modifies the speckles deforms with the changing of the observing angle. This phenomenon can be seen in Fig. $7 \mathrm{a}-\mathrm{f}$ where the letter ' $\mathrm{L}$ ' becomes thinner when the observing point moves from point ' $a$ ' to point ' $\mathrm{f}$ ' as demonstrated in Fig. $7 \mathrm{~g}$. Correspondingly, the calculated object size from Equation (6) will also change with the changing of the observing point. Nevertheless, if the observing angle is known, the deviation can be compensated.
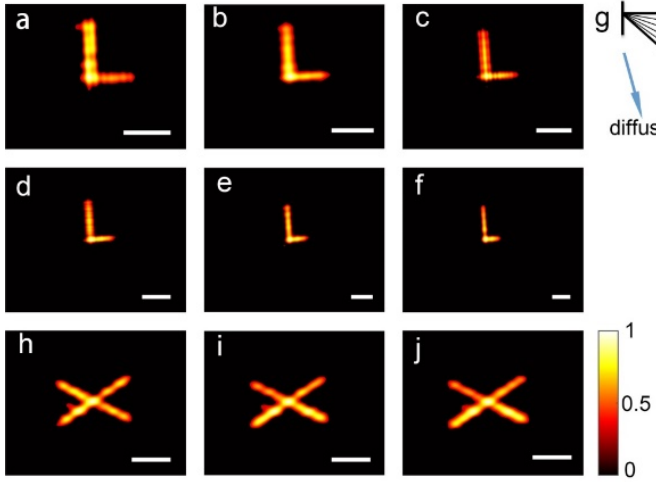

Fig. 7. Demonstration of the AFOV and the depth of field (DOF). (a)-(f) The images reconstructed from the raw images taken at the observing angles from $0^{\circ}$ to $45^{\circ}$ respectively. The power of the laser was adjusted to ensure the sufficient signal intensity. (g) Experiment setup shows measuring angles: letters a to findicate the camera positions where the raw images for figures (a)-(f) were taken. The image distance along the optical axis was $10 \mathrm{~cm}$ and the camera was moved away from the optical axis from $0 \mathrm{~cm}$ to $12.5 \mathrm{~cm}$ with an increment of $2.5 \mathrm{~cm}$. (h)-(j) are reconstructed object images with the object distances of $10 \mathrm{~cm}, 20$ $\mathrm{cm}$, and $30 \mathrm{~cm}$ respectively. The image distance was $10 \mathrm{~cm}$. Scale bars are $2.5 \mathrm{~mm}$. The width of object ' $\mathrm{L}$ ' is $2.5 \mathrm{~mm}$ and that of object ' $\mathrm{X}$ ' is 5 $\mathrm{mm}$.

Fig. 7(h)-(j) show the reconstructed images with different object distance of $10 \mathrm{~cm}, 20 \mathrm{~cm}$, and $30 \mathrm{~cm}$ respectively. It is clearly shown that the object distance does not influence the quality of the reconstructed object images, provided that the speckle size satisfies the Nyquist criteria and the diffraction from the object can be ignored. This suggests that the proposed imaging system is not limited by the focal length. When collimated light is used as the illumination source, the DOF of our proposed imaging system is not restricted when the diffraction of the object can be ignored, as demonstrated in Fig. 7h-j. When non-collimated light is used, the object cannot be too small because the projections of adjacent regions of the object may overlap. In our experiment the object can be reconstructed without perceivable quality loss when the object distance was up to $30 \mathrm{~cm}$ with collimated illumination (Fig. 7(j)). Therefore, with large FOV and deep DOF (or a long focal length) this system can be regarded as a flexible lensless imaging system.

\section{E. Non-invasive imaging}

In non-invasive biomedical imaging applications, such as imaging through body tissues, the light transmits twice through the diffusing material. This makes the object reconstruction more challenging. Firstly, we show that our method is suitable for biomedical imaging applications by using the system shown in Fig. 8a, where the object is sandwiched between two diffusers. Fig. 8b-Fig. 8d show the reconstructed results when the object distances (marked with ' $\mathrm{D}$ ' in Fig. 8a) were $7.5 \mathrm{~cm}, 5 \mathrm{~cm}$ and $2.5 \mathrm{~cm}$ respectively. It can be seen that the quality of the reconstructed image is improved for a smaller $\mathrm{D}$, owing to the reduced overlapping of the diverging beams from different regions of the object.
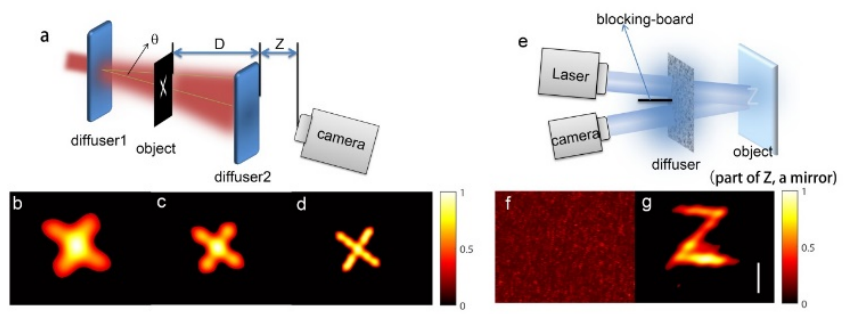

Fig. 8. Experiments for Non-invasive imaging. (a) Experimental setup. (b)-(d) The letter $\mathrm{x}$ ( $4 \mathrm{~mm}$ in height) was imaged from different object distances of $7.5 \mathrm{~cm}, 5 \mathrm{~cm}$, and $2.5 \mathrm{~cm}$. Diffuser 1 was ground glass and diffuser 2 was a piece of paper. (b)-(d) show the reconstructed objects. (e)-(g) Proof-of-Concept experiment for non-invasive imaging. (e) Experimental setup with collimated laser illumination. The blocking board was used to isolate the light reflected from the diffuser. (f) The raw camera image. (g) The reconstructed object image from (f). The height of object ' $\mathrm{X}$ ' is $4 \mathrm{~mm}$ and that of object ' $\mathrm{Z}$ ' is $5 \mathrm{~mm}$. In (b)-(d) the reconstructed object images contain $518,428,365$ pixels respectively in vertical direction. Scale bar in (g) is $2.5 \mathrm{~mm}$.

We then tested the feasibility of using this method to reconstruct object images through highly-scattering biological tissues. In our experiment, a piece of fruit tissue (a pear slice with a thickness approximately $1 \mathrm{~mm}$ ) was used as the scattering material, and is thick enough to block the details of the object under room-light condition. A mirror covered by a ' $Z$ ' shaped aperture was used as the object. The experimental setup is shown in Fig. 8e. In this case, the laser for imaging the ' $Z$ ' aperture was scattered twice by the pear slice, similar to biomedical imaging applications where the target is underneath biotissues. Because of the highly diffusive nature of the pear slice, the intensity of the light drops sharply after the light is scattered twice by the pear slice. Therefore we chose a mirror covered by an aperture as the object to enabling more light reaching the camera. An alternative option is to use a camera with high sensitivity and low noise. The object image (Fig. 8g) was reconstructed with our reconstruction method from the raw speckle image shown in Fig. 8f.

In Non-invasive imaging setup shown in Fig. 8a, the illumination has a divergence angle. If we use $\theta$ to denote the angle of divergence of the beam after passing Diffuser 1 in Fig. 8a, Equation 6 can be modified to:

$$
X=\frac{\lambda \cdot Z}{H+2 \cdot f \cdot \tan \theta} .
$$

Combining Equation 5 and Equation 7, we obtain: 


$$
H=\frac{\lambda \cdot Z \cdot N}{L \cdot A}-2 \cdot D \cdot \tan \theta
$$

where $\mathrm{D}$ is the distance from the object to Diffuser 2 . Equation 8 means that the size of the object can only be estimated roughly, if the angle of divergence of the beam from Diffuser 1 and the distance between the object plane and Diffuser 2 are unknown. This challenge needs to be addressed for further practical applications.

\section{DISCUSSION}

Our proposed method for image reconstruction through turbid media is based on the demodulation of the object shape from objectmodulated speckles. Therefore, it is crucial that individual speckles are sufficiently sampled. The minimum sampling requirement, according to Nyquist Theorem, requires the size of a single speckle to be at least the size of two camera pixels. Clearly, bigger speckles preserve the fine details of the object. At the same time, however, too big speckles may result in that the object image cannot be reconstructed as the high frequency components of the object may be lost.

It is also worth noting that when the object is too small, the diffraction effect becomes dominant and the projections of different regions of the object on the diffuser may overlap. This may lead to unsuccessful image reconstructions. This may also occur when the illumination is diverged, such as the experiment described in the experiment section of 'Non-invasive imaging'. However, in our experiments, the distance between the object and the scattering medium can be up to $5 \mathrm{~cm}$ for successful imaging reconstruction. Besides, by adding a lens between the camera and the second diffuser in Fig. 7a followed by a careful focusing, the object can be reconstructed because of the curtain-shower effect proposed by $\mathrm{E}$. Edrei [31].

The resolvable size, both the minimum and the maximum, is impacted by many factors. From the sampling aspect, the maximum object size, when the illumination is collimated, is determined by the Nyquist sampling frequency, i.e. $H \leq \frac{\lambda \cdot Z}{2 \cdot X}$. When the illumination is diverged, the maximum detectable size is also affected by divergence angle. The minimum resolvable object size, according to Equation (2) and Fig. 2(g), is determined by the background noise level. The intensity of the background noise need to be low enough that the detailed power spectrum of the object can be extracted. The level of background noise is proportional to the speckle number enrolled in the reconstruction. Therefore many methods take multiple speckle images for image reconstruction. Supposing $N^{\prime}$ is the minimum number of speckles that produces background noise lower than the intensity of the power spectrum of the object, then the minimum resolvable object can be calculated from $H \geq \frac{N^{\prime} \cdot \lambda \cdot Z}{L^{2}}$. Therefore the resolvable object range is $\frac{N^{\prime} \cdot \lambda \cdot Z}{L^{2}} \geq H \leq \frac{\lambda \cdot Z}{2 \cdot X}$.

The resolution of this method is also affected by the scattering properties and the thickness of the diffuser, which finally determines the intensity at different angles after the diffusion. This can be estimated by $l_{t} / l$ with $l_{t}$ denoting transport mean free path and $l$ denoting the thickness of the diffuser $[45,46]$. When $l_{t} / l<1$, photon diffusion is weak then we can estimate that the resolution is diffraction limit with an extreme situation of zero thickness of the diffuser. When $l>l_{t}$, the accuracy of this method decreases. The line shape of a point object after the diffuser need to be calculated [47] and the real resolution due to the scattering effect can be estimated.

In applications when the diffuser has movement, the recorded speckles may blur due to the exposure duration of the camera. The accuracy of the reconstruction is consequently affected. In this case, decreasing the exposure time according to the moving speed of the diffuser and using a low noise camera can be helpful. Alternatively changing the $\mathrm{CW}$ to pulsed illumination is also an option.

Theoretically speaking, the speckle image contains the grey scale information of the object, but the phase retrieval algorithm in the object reconstruction introduces intensity distribution bias, which can be noticed in the figures in this paper. Therefore the grey scale cannot be stably recovered. A potential method to break this limit is under investigation.

Funding Information. This work was sponsored by the open project of State Key Laboratory of Quantum Optics and Quantum Optics Devices, Shanxi University (KF201605), National Natural Science Foundation of China (NSFC) $(61605092,11474169)$ and Science and Technology Innovation Program of the Chinese Academy of Sciences. This work was supported by the Engineering and Physical Sciences Research Council [grant number EP/L022559/2].

\section{References}

1. S. M. Kolenderska, O. Katz, M. Fink, and S. Gigan, "Scanning-free imaging through a single fiber by random spatio-spectral encoding," Optics letters 40, 534-537 (2015).

2. M. Gehm, R. John, D. Brady, R. Willett, and T. Schulz, "Single-shot compressive spectral imaging with a dual-disperser architecture," Optics Express 15, 14013-14027 (2007).

3. C.-L. Hsieh, Y. Pu, R. Grange, G. Laporte, and D. Psaltis, "Imaging through turbid layers by scanning the phase conjugated second harmonic radiation from a nanoparticle," Optics Express 18, 20723-20731 (2010).

4. A. Gatti, E. Brambilla, M. Bache, and L. A. Lugiato, "Ghost imaging with thermal light: comparing entanglement and classicalcorrelation," Physical review letters 93, 093602 (2004).

5. R. E. Meyers, K. S. Deacon, and Y. Shih, "Turbulence-free ghost imaging," Applied Physics Letters 98, 111115 (2011).

6. X.-H. Chen, Q. Liu, K.-H. Luo, and L.-A. Wu, "Lensless ghost imaging with true thermal light," Optics letters 34, 695-697 (2009).

7. Y.-K. Xu, W.-T. Liu, E.-F. Zhang, Q. Li, H.-Y. Dai, and P.-X. Chen, "Is ghost imaging intrinsically more powerful against scattering?," Optics Express 23, 32993-33000 (2015).

8. A. K. Singh, D. N. Naik, G. Pedrini, M. Takeda, and W. Osten, "Looking through a diffuser and around an opaque surface: A holographic approach," Optics express 22, 7694-7701 (2014).

9. W. Harm, C. Roider, A. Jesacher, S. Bernet, and M. Ritsch-Marte, "Lensless imaging through thin diffusive media," Optics Express 22, 22146-22156 (2014).

10. S. Li, and J. Zhong, "Dynamic imaging through turbid media based on digital holography," JOSA A 31, 480-486 (2014).

11. E. Leith, C. Chen, H. Chen, Y. Chen, D. Dilworth, J. Lopez, J. Rudd, P.-C. Sun, J. Valdmanis, and G. Vossler, "Imaging through scattering media with holography," JOSA A 9, 1148-1153 (1992).

12. W. Xu, M. H. Jericho, I. A. Meinertzhagen, and H. J. Kreuzer, "Digital inline holography of microspheres," Applied Optics 41, 5367-5375 (2002).

13. N. Verrier, S. Coëtmellec, M. Brunel, and D. Lebrun, "Digital in-line holography in thick optical systems: application to visualization in pipes," Applied Optics 47, 4147-4157 (2008).

14. C. Graulig, M. Kanka, and R. Riesenberg, "Phase shifting technique for extended inline holographic microscopy with a pinhole array," Optics Express 20, 22383-22390 (2012).

15. J. S. Tyo, "Enhancement of the point-spread function for imaging in scattering media by use of polarization-difference imaging," Journal of the Optical Society of America A 17, 1-10 (2000).

16. N. Shashar, S. Sabbah, T. W. C. Tyo, J. S, M. P. Rowe, E. N. Pugh, and N. Engheta, "Target detection in optically scattering media by polarizationdifference imaging," Applied Optics 35, 1855-1870 (1996). 
17. S. P. Morgan, P. K. Man, and M. G. Somekh, "Effects of polarization state and scatterer concentration on optical imaging through scattering media," Applied Optics 36, 1560-1565 (1997).

18. A. P. Mosk, A. Lagendijk, G. Lerosey, and M. Fink, "Controlling waves in space and time for imaging and focusing in complex media," Nature photonics 6, 283-292 (2012).

19. M. Nixon, O. Katz, E. Small, Y. Bromberg, A. A. Friesem, Y. Silberberg, and N. Davidson, "Real-time wavefront shaping through scattering media by all-optical feedback," Nature Photonics 7, 919-924 (2013).

20. Y. Guan, O. Katz, E. Small, J. Zhou, and Y. Silberberg, "Polarization control of multiply scattered light through random media by wavefront shaping," Optics letters 37, 4663-4665 (2012).

21. O. Katz, E. Small, and Y. Silberberg, "Looking around corners and through thin turbid layers in real time with scattered incoherent light," Nature Photonics 6, 549-553 (2012).

22. R. Horstmeyer, H. Ruan, and C. Yang, "Guidestar-assisted wavefrontshaping methods for focusing light into biological tissue," Nature Photonics 9, 563-571 (2015).

23. I. M. Vellekoop, "Feedback-based wavefront shaping," Optics Express 23, 12189-12206 (2015).

24. Y. L. Hasan, W. L. Vos, and A. P. Mosk, "Optimal control of light propagation through multiple-scattering media in the presence of noise," Biomedical Optics Express 4, 1759-1768 (2013).

25. I. I. Freund, M. Rosenbluh, and S. Feng, "Memory effects in propagation of optical waves through disordered media," Physical Review Letters 61, 2328-2331 (1988).

26. B. Jacopo, E. G. Putten, Van, B. Christian, L. Ad, W. L. Vos, and A. P. Mosk, "Non-invasive imaging through opaque scattering layers," Nature 491, 232-234 (2012).

27. O. Katz, P. Heidmann, M. Fink, and S. Gigan, "Non-invasive single-shot imaging through scattering layers and around corners via speckle correlations," Nature Photonics 8, 784-790 (2014).

28. J. W. Goodman, "Dependence of image speckle contrast on surface roughness," Optics Communications 14, 324-327 (1975).

29. J. D. Briers, and S. Webster, "Laser speckle contrast analysis (LASCA): a nonscanning, full-field technique for monitoring capillary blood flow," Journal of biomedical optics 1, 174-179 (1996).

30. D. A. Boas, and A. K. Dunn, "Laser speckle contrast imaging in biomedical optics," Journal of biomedical optics 15, 011109-011109-011112 (2010).

31. E. Edrei, and G. Scarcelli, "Optical imaging through dynamic turbid media using the Fourier-domain shower-curtain effect," Optica 3, 71-74 (2016).

32. M. Saito, H. Okayama, K. Inoue, T. Yoshii, G. Hiasa, T. Sumimoto, K. Nishimura, A. Ogimoto, and J. Higaki, "Carotid arterial circumferential strain by two-dimensional speckle tracking: a novel parameter of arterial elasticity," Hypertens Res 35, 897-902 (2012).

33. P. S. Idell, J. R. Fienup, and R. S. Goodman, "Image synthesis from nonimaged laser-speckle patterns," Optics Letters 12, 858-860 (1987).

34. P. S. Idell, J. D. Gonglewski, D. G. Voelz, and J. Knopp, "Image synthesis from nonimaged laser-speckle patterns: experimental verification," Optics Letters 14, 858-860 (1989).

35. D. G. Voelz, J. D. Gonglewski, and P. S. Idell, "Image synthesis from nonimaged laser-speckle patterns: comparison of theory, computer simulation, and laboratory results," Applied Optics 30, 3333-3344 (1991).

36. M. Elbaum, M. King, and M. Greenbaum, "Laser correlography: Transmission of high-resolution object signatures through the turbulent atmosphere," Nasa Sti/recon Technical Report N 76 (1974).

37. S. J. Kirkpatrick, D. D. Duncan, and E. M. Wells-Gray, "Detrimental effects of speckle-pixel size matching in laser speckle contrast imaging," Optics Letters 33, 2886-2888 (2008).

38. A. Apostol, and A. Dogariu, "Spatial correlations in the near field of random media," Physical Review Letters 91, 9105-9117 (2003).

39. R. Carminati, "Subwavelength spatial correlations in near-field speckle patterns," Physical Review A 81, 1532-1532 (2010).

40. J. R. Fienup, "Phase retrieval algorithms: a comparison," Applied Optics 21, 2758-2769 (1982).
41. S. Marchesini, H. He, H. N. Chapman, S. P. Hau-Riege, A. Noy, M. R. Howells, U. Weierstall, and J. C. Spence, "X-ray image reconstruction from a diffraction pattern alone," Physical Review B 68, 140101 (2003).

42. S. J. Kirkpatrick, D. D. Duncan, R. K. Wang, and M. T. Hinds, "Quantitative temporal speckle contrast imaging for tissue mechanics," Journal of the Optical Society of America A 24, 3728-3734 (2007).

43. S. J. Kirkpatrick, D. D. Duncan, and E. M. Wells-Gray, "Detrimental effects of speckle-pixel size matching in laser speckle contrast imaging," Optics Letters 33, 2886-2888 (2008).

44. J. W. Goodman, "Statistical properties of laser speckle patterns," Laser Speckle \& Related Phenomena 9, 9-75 (1975).

45. V. Ntziachristos, "Going deeper than microscopy: the optical imaging frontier in biology," Nature Methods 7, 603-614 (2010).

46. J. Bingi, M. Hemalatha, R. W. Anita, C. Vijayan, and V. M. Murukeshan, "Asymmetric transmission and optical low-pass filtering in a stack of random media with graded transport mean free path," Optical Materials 49, 15-20 (2015).

47. E. Akkermans, P. E. Wolf, and R. Maynard, "Coherent backscattering of light by disordered media: Analysis of the peak line shape," Physical Review Letters 56, 1471-1474 (1986). 